\title{
Juvenile Dermatomyositis: A Case Report
}

\author{
Bhuiyan $\mathrm{I}^{1}$, Hossain $\mathrm{MS}^{2}$, Akhter $\mathrm{M}^{3}$, Chowdhury $\mathrm{SR}^{4}$
}

\begin{abstract}
Juvenile dermatomyositis is a chronic inflammatory multisystem disease that affects primarily the skin and muscle. A 5 years old girl presented with heliotrope rash, gottron's papules, and muscular weakness for one and half month. Other systemic examination revealed no abnormalities. Investigation reveals- Erythrocyte Sedimentation rate was raised, muscle enzymes were several fold elevated, skin biopsy consistent with dermatomyositis, EMG consistent with polymyositis. So confirmed diagnosis was made as Juvenile dermatomyositis (JDM). The patient was managed by systemic steroid and showed good response to treatment. [J Shaheed Suhrawardy Med Coll, 2013;5(1):63-66]
\end{abstract}

Key words: Juvenile dermatomyositis, heliotrope rash, gottron's papules.

Received: February 2013; Revised: March 2013; Accepted: May 2013

\section{Introduction}

The invagination of a segment of the intestine into the Childhood/ Juvenile dermatomyositis (JDM) is a chronic inflammatory multisystem disease affects primarily the skin and muscle $e^{1,2,5,6}$.

Dermatomyositis have two peaks of occurrence, one in childhood and one between the age of 45 and 65 years ${ }^{3,5}$. The estimated annual incidence rates ranged from 2.5 to 4.1 cases per million children ${ }^{1,2}$.

JDM is autoimmune in pathogenesis and results from a vasculopathy. Both cell mediated immunity to muscle antigen and immune complex disease may play role in the pathogenesis $^{6}$.

The diagnosis of dermatomyositis depends on fulfillment of criteria established by Bohan and Peter, which include one of the typical rashes, as well as 3 of the 4 following ${ }^{1,3,6}$.

i) Symmetrical proximal muscle weakness

ii) Electromyography evidence of an inflammatory myopathy

iii) Elevated serum muscle enzymes

$1 v$ ) inflammatory myositis on muscle biopsy.
Skin manifestations of dermatomyositis include heliotrope rash, periorbital oedema, gottron's papule \& Gottron's sign, erythematous malar rash, confluent macular violaceous erythema overlying the extensor aspect of the upper extremity, $\mathrm{V}$ area of anterior neck and chest, central aspect of the face, peri orbital areas, forehead of the scalp, lateral aspect of the hip and thigh, periungual telangiectasia, poikiloderma, hyperkeratosis or mechanical hands, cuticular over growth, panniculitis, cutaneous vasculitis and scalp involvement including alopecia ${ }^{1-3,5}$.

Gottron sign and Gottron papules are pathognomic of the JDM, whereas heliotrope rash, oedema and grossly visible periungual telangiectasia associated with dystrophic cuticles are highly characteristic.

Two types of childhood variants exist.Brunsting type, more common, have a slow course, progressive weakness, calcinosis, and responsiveness by steroid. The other one is Banker type, associated with a vasculitis of the muscles and gastro-intestinal tract, rapid onset of severe weakness, steroid unresponsiveness and high death rate ${ }^{3}$.

1. Dr. Ishrat Bhuiyan, Assistant Professor, Department of Dermatology \& Venereology, Shaheed Suhrawardy Medical College, Dhaka

2. Dr. Md. Shahadat Hossain, Assistant Professor, Department of Dermatology \& Venereology, Shaheed Suhrawardi Medical College, Dhaka

3. Dr. Mafuza Akhter, Assistant Professor, Department of Dermatology \& Venereology, Shaheed Suhrawardy Medical College, Dhaka

4. Dr. Shaheen Reza Chowdhury, Assistant Professor, Department of Dermatology \& Venereology, Shaheed Suhrawardy Medical College, Dhaka

Correspondence:

Dr. Md. Shahadat Hossain, Assistant Professor, Department of Dermatology \& Venereology, Shaheed Suhrawardy Medical College, Dhaka, Bangladesh; Email: dr.shahadat @ hotmail.com; Cell No.: +8801819292667

Conflict of interest: No conflict of interest

Contributions by authors: All authors contributed in the management of case. 
Childhood type differs from the adult type by the presence of vasculitis of small blood vessels, which can involve the intestinal tract and myocardium, besides skin and muscle Cal cinosis present in childhood variant absent in adul thood variant. Childhood variant rarely associated with malignancy. Adulthood variant commonly associated with mal ignancy, childhood variant responsive to treatment and if diagnosed early and treated vigorously. The great majority of the affected children make good recovery ${ }^{2,3}$.

\section{Case Presentation}

In 10th J une 2012, a 5 years old female child presented to the Department of Dermatology and Venereology, Shaheed Suhrawardy Medical College \& Hospital, Dhaka, Bangladesh with the complaints of gradual swelling of eyelids, rashes over different parts of the body for one and half months, gradual weakness and bodyache for one month.

On examination there was peri orbital oedema with heliotrophic rash.Confluent, macular violaceous erythema involving the lower part of face, $V$ area of neck, chest, sides of the neck, extensor aspect of the upper extremities, back and extensor aspect of the kneejoints.

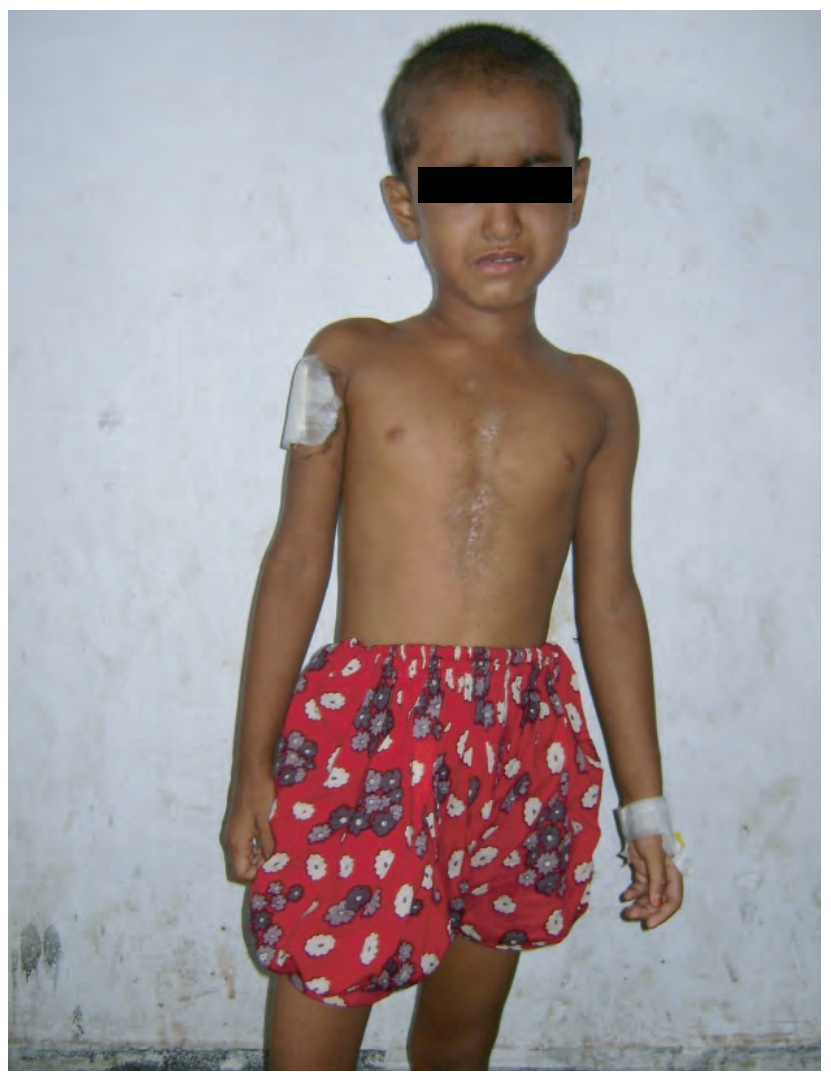

Figure I: Five years old girl presented with skin rash on different parts of the body and muscular weakness.

On the extensor surface of the small joints of both hands, there was erythematous, flat topped papular lesion (Gottron's papule).

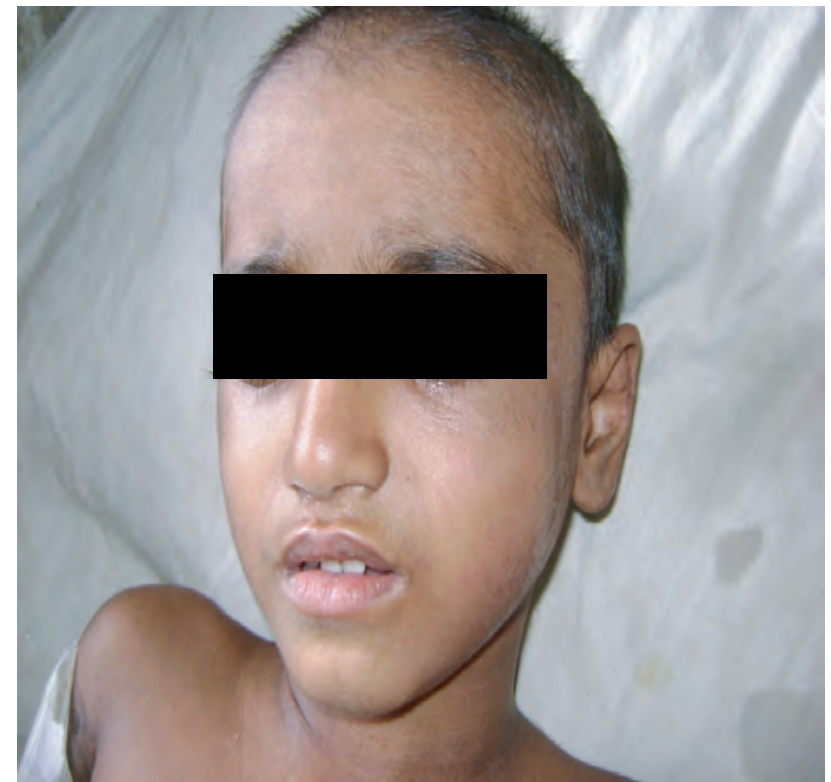

Figure II: Heliotrope rash with confluent macular violaceous erythema involving the face.

In the upper back and central chest there was mottled hyper and hypopigmentation, atrophy \& telangiectasiapoikiloderma. Investigation revealed ESR-55mm in Ist hour, $\mathrm{Hb} \%-14$ gm/dl, WBC-10000/cc, Neutrophil-72\%, Lymphocyte-26\%, Eosinophil-2\%, ANA- Negative, Anti ds DNA-Negative. Muscle enzymes reveals serum CPK $4905 \mathrm{u} / \mathrm{L}$, skin AST (SGOT)-200 u/L, Serum Aldolase 19.2U/L, Serum LDH-138 U/L. Histopathological examination reveals hyperkeratosis, keratotic plugging, mild atrophy of the epidermis, basal liquefaction and lympho histiocytic infiltration at dermo-epidermal junction as well as peri vascular region in the upper dermis. Diagnosis was consistent with Dermatomyositis.

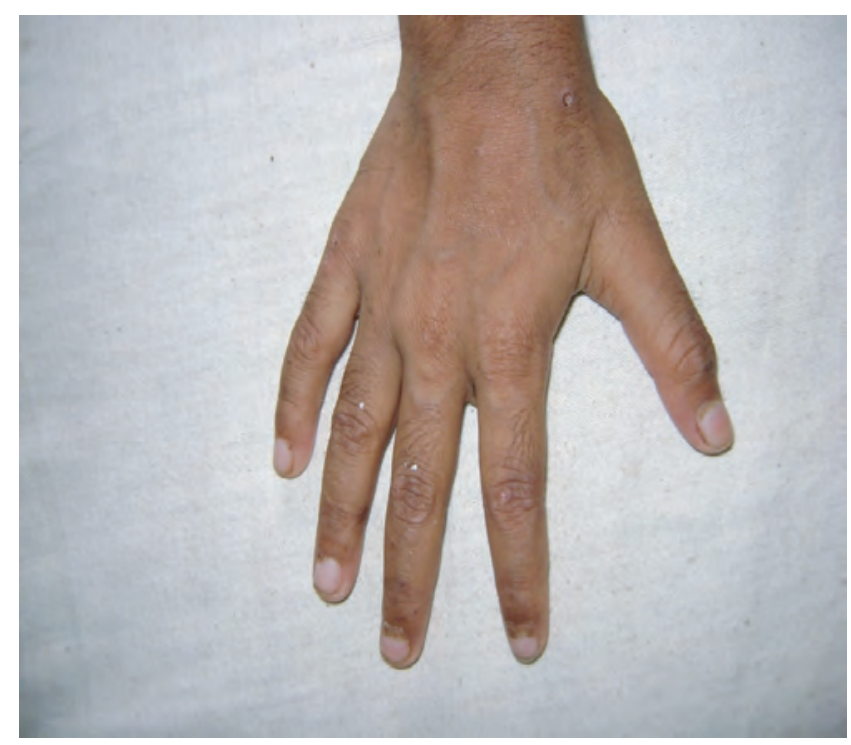

Figure III: Erythematous flat topped papular lesion over the extensor aspect of the small joints of the hand (Gottron's papule). 


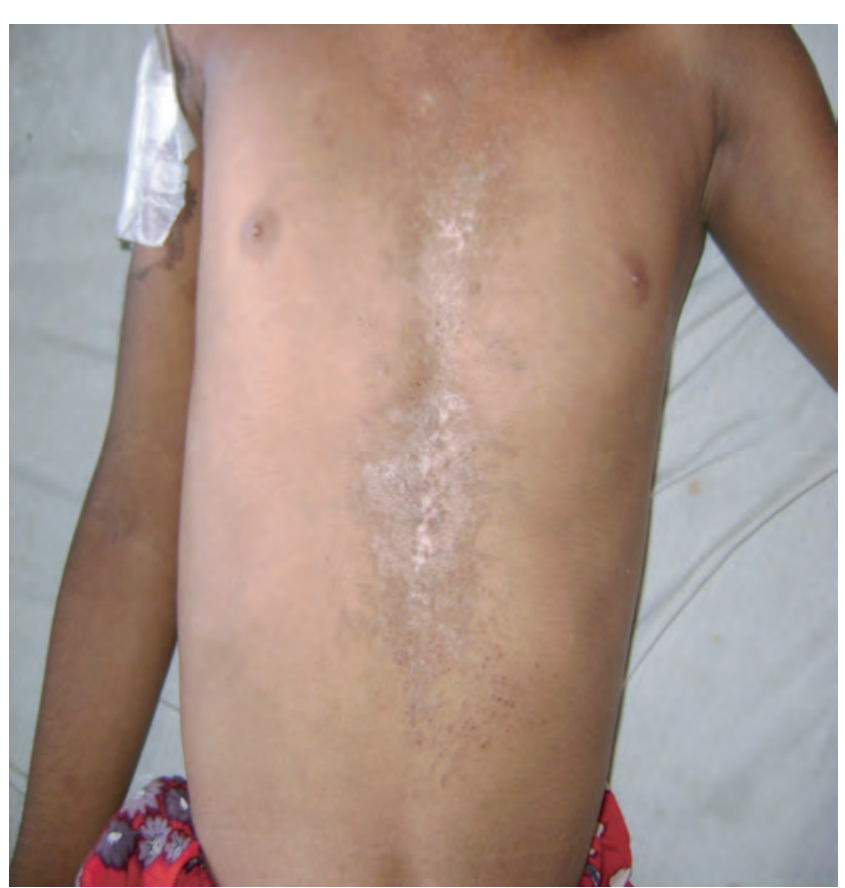

Figure IV: Mottled hyper and hypopigmentation, atrophy and telangiectasia involving in the central part of chest and upper abdomen (poikilodermatous change).

Electromyogram was consistent with polymyositis. All other relevant investigations were normal. So, based on clinical features and investigation done, the patient was diagnosed as a case of juvenile dermatomyositis. The patient was treated with systemic steroid and other supportive measure. At the time of discharge there was a clear response to treatment with resolution of the skin lesion and gradual improvement of muscular weakness and musclestrength. She was advised for regular follow up.

\section{Discussion}

J uvenile dermatomyositis (JDM) is a rare multisystemic disease and auto immune in origin. Environmental factors

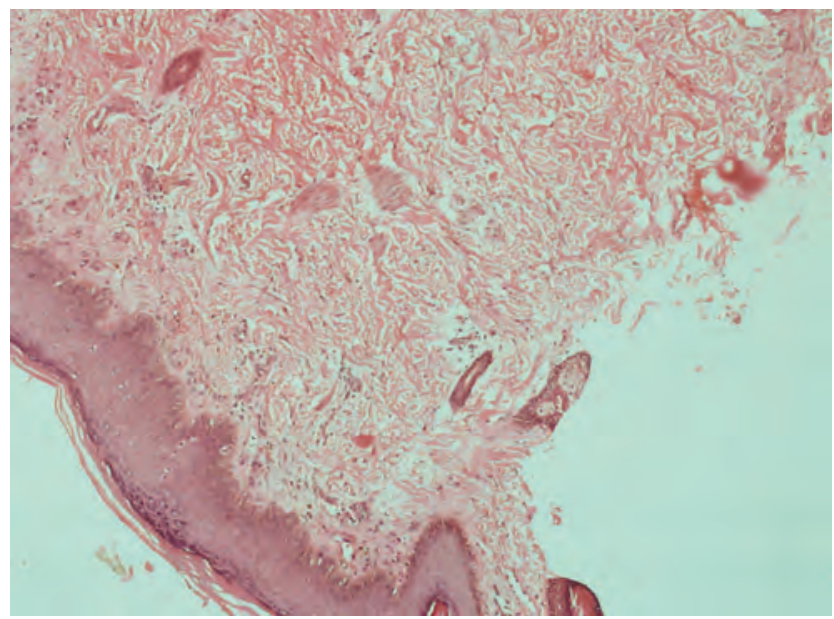

like Coxsackie B virus, Parvo virus B-19, Epstein-Barr Virus (EBV), Toxoplasmosis, Staphylococcal osteomyelitis and arthiritis, Staphylococcal infection, HIV may act as triggering factors ${ }^{2-4,7}$.

Increased incidence of HLA- B8 in JDM in white people4. The disease usually has an insidious onset. The proximal muscle groups are classically more affected than the distal groups, exhibiting progressive weakness and often tendemess in symmetrical distribution ${ }^{7,8}$.

For definite diagnosis, Gottron's papule and heliotrope rash are pathognomic of the disease Calcinosis is one of the hallmarks of JDM ${ }^{7,10}$.

The electron microscopic examination reveal earliest change in the muscle is endothelial cell damage with swelling, cell necrosis and regeneration ${ }^{3,8}$.

DIF findings reveal granular deposition of immunoglobulin and $c 3$ in the intima of the blood vessel ${ }^{1-3}$.

Muscle weakness and secondary contractures are in inevitable part of JDM. Vigorous physiotherapy has paramount importance in minimizing permanent contracture, 10 .

Those who did not respond to corticosteroid, methotrexate, hydroxychloroquine, azathioprine and intravenous immunoglobulin, mycophenolate mofetil were use ${ }^{2,4}$.

The early aggressive management improved outcome $e^{1,3}$, and $^{11}$.

\section{Conclusion}

Juvenile dermatomyositis (JDM) appears to be a different disease from adult dermatomyositis. Before the steroid therapy prognosis was bad, but early aggressive steroid therapy prognosis is good. Prognosis is variable and related to the degree of vasculitis.

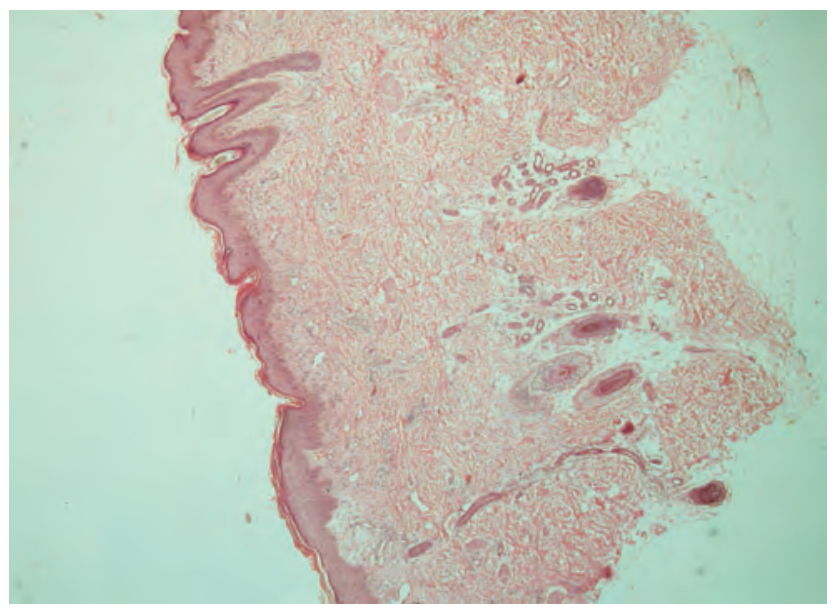

Figure 5a and 5b: Histopathology slide showing hyperkeratosis, keratotic plugging, basal cell liquefaction and lymphohistiocytic infiltration in to the upper dermis. 


\section{R eferences}

1. Fisler RE, Liang MG, Fuhlbrigge RC, Yalcindag A, Sundel RP. Aggressive management of J uvenile dermatomyositis results in improved outcome and decreased incidence of calcininosis. J Am Acad Dermatol 2002;47(4):505-511

2. Richard D.Sontheimer, Christopher B. Hansen and Melissa I Costner. Dermatomyositis. Fitz Patricks Dermatology in general medicine. Edn 8th, 2012.1926-1942.

3. William D. James, Timothy G. Berger, Dirk M. Eloston,. Andrews's diseases of the skin. 11th edition 2011, 165-168.

4. M.J.D. Goodfield, S.K. Jones and D.J.Veale, the 'Connective Tissue Diseases'. Rook's Textbook of Dermatology.8thedition ,2010;51.12051.129

5. Harry Winfield, Christine Jaworsky.Connective Tissue Diseases. Lever's Histopathology of the skin.10th edition, 2009, 293-295.

6. Adriana Maluf Elias Sallum, Maria Helena Bittencurt Kiss, Silvana Sachetti, Maria Bernadate Dutra Resende, Kelly Cristina Moutinho, Mary de Souza Carvalho, Clovis Arthur Almeida Silva, Suely Kazue NagahashiMarieJ uvenile dermattomyositis: clinical, Iaboratorial, histological, therapeutical and evolution parameters of 35 patients Arq Neuropsiquiatr 2002;60(4): 889-899.

7. Geetha chari, Teresita A, Lavde Juvenile dermatomyosits; a review international Pediatrics .vol-15: No.1 2000.

8. Lisa G, Rider MD.Cal cinosis in J uvenile dermatosis; Pathogenesis and Current therapies. Paediatric Rheumatology On line J ournal. 2002; 1-15.

9. Chiv SK, Yang YH, Wang LC, Chiang BL. Ten year experience of Juvenile Dermatomyositis: A retrospective study. Journal Microbial Immunol Infection. 2007 Feb; 40(1); 68-70.

10. Shehata R, al -Mayouf S, al-Dalna S. Juvenile Dermatomyositis; Clinical profile and disease course in 25 patients.Clin Exp Rheumatol, .1999.J an-Feb; 17(1): 115-118.

11. Tamada Y, Yokochi K, Nitta Y, et al. Lichen planus pemphigoides: identification of $180 \mathrm{KD}$ hemidesmosome antigens. J AmAcad Dermatol 1995:32: 883-887.

12. NakagawaT, TakaiwaT. Cal cinosis cutis in J uvenile Dermatomyositis response to aluminium hydroxide treatment. J Dermatology 1993Sept,20(9):558-560 